\title{
Sugar intake and craving during alcohol withdrawal in alcohol use disorder inpatients
}

\author{
ALARCON Régis ${ }^{1}$, TIBERGHIEN Margaux ${ }^{1}$, TROUILLET Raphael ${ }^{2}$, PELLETIER Stéphanie ${ }^{1}$, LUQUIENS \\ Amandine $^{1,3}$, AHMED Serge $\mathrm{H}^{4}$, NALPAS Bertrand ${ }^{1,5}$, ALAUX-CANTIN Stéphanie $^{6}$, NAASSILA Mickaël ${ }^{6}$, \\ PERNEY Pascal ${ }^{1,7}$
}

\section{Adresses}

${ }^{1}$ Addictions Department, CHU Caremeau, Place du Pr R. Debré, 30029 Nîmes, France ${ }^{2}$ Université Paul Valery-Montpellier 3, Montpellier

${ }^{3}$ Paris-Saclay University, Univ. Paris-Sud, UVSQ, CESP, INSERM, Villejuif, France

${ }^{4}$ IMN - UMR 5293 - Université de Bordeaux, 146 rue Léo-Saignat, 33076 Bordeaux cedex - France

${ }^{5}$ Department of Scientific Information and Communication (DISC), Inserm, 101 rue de Tolbiac, 75013 Paris, France

${ }^{6}$ Inserm UMR1247, Groupe de Recherche sur l'Alcool et les Pharmacodépendances (GRAP), Université de Picardie Jules Verne, Faculté de Pharmacie, 1 rue des Louvels, Amiens, France

${ }^{7}$ Inserm U1018, Hôpital Paul Brousse, 16 avenue Paul Vaillant Couturier, 94800 Villejuif, France

\section{Correspondence and offprints}

Bertrand Nalpas, MD, PhD

Service d'Addictologie, CHU Caremeau

Place du Pr R. Debré

30029 Nîmes, France

Tel: +33 (0)4 66687214

E-mail: bertrand.nalpas@inserm.fr

Running head: Sugar intake and abstinence

Word count: 2440

Number of figures and tables: 3 tables and 2 figures

No conflicts of interest for this study 


\section{Abstract}

Addiction transfer from alcohol to sugar has been demonstrated in the alcohol postdependent state model in rats. To assess whether changes in sugar intake and craving can occur during alcohol withdrawal in humans we conducted a prospective, observational study in a university hospital addictions treatment center. Recruited patients had severe alcohol use disorder, were hospitalized for 7 days in the short-stay unit for alcohol withdrawal and then for 6 weeks in the rehabilitation unit. During the hospital stay, they had no access to alcohol but had full access to sweet products and beverages in a shop and vending machines located inside the hospital.

Alcohol craving was assessed using a visual analogue scale on days 1, 15 and 45 . Sugar craving, sweet products stored by patients in their rooms and weight were assessed on the same days.

Thirty-five patients were included. Sugar craving increased in 14 patients during the hospital stay while no change was observed in the remaining 21 . Significant increases in both the amounts of sweet products stored in the patients' rooms $(p<0.02)$ and weight $(p<0.05)$ were observed only in the sugar craving group. During the same period, alcohol craving decreased significantly in all patients. Changes in tobacco smoking were not different according to the sugar craving status and therefore cannot explain the observed differences.

In conclusion, increased intake and craving for sugar after alcohol withdrawal were observed in $40 \%$ of the patients included in our prospective study and these results were similar to those of a study conducted in the alcohol post-dependent state model in rats.

Keys words: alcohol use disorder, craving, sugar, substitutability, withdrawal 


\section{INTRODUCTION}

Alcohol Use Disorder (AUD) is a chronic, recurrent disease for which two care objectives are currently accepted: a reduction in alcohol intake and abstinence. ${ }^{1}$ After alcohol withdrawal, some patients with AUD report significant weight gain and an attraction to highly sweet tastants. ${ }^{2}$ This may explain the sweet products stored by some inpatient in their rooms during withdrawal. Similarly, AUD outpatients frequently describe dietary changes and the emergence of a strong craving for sweet products after they stop consuming alcohol.

Several studies have shown that increased sugar intake causes brain neurochemistry changes similar to those observed after consumption of addictive drugs ${ }^{3,4}$. Palatable foods with a high sugar or fat content affect dopamine, opioid, and serotoninergic receptors in the brain ${ }^{4}$. Sugar consumption has been linked to use of addictive substances, and individuals dependent on alcohol or drugs have been found to have a stronger sweet preference than nondependent individuals $\mathrm{s}^{5,6,7}$. There are also studies showing that high sugar intake and sweet preference are associated with higher substance use $\mathrm{e}^{8,9,10}$. In their twin study, Treur et al. (2016) $)^{10}$ showed that genetic factors explained approximately $50 \%$ of the association between sugar intake (through drinks) and polysubstance use.

After drug withdrawal, neurobiological and neuroimaging data suggest that there may be an overlap between drug and food craving and addiction transfer ${ }^{11}$. For example, in an American qualitative study examining interviews with 25 patients, it appeared that after recovery from substance addiction, some patients reported weight gain, binge eating and use of food as a substitute or to regulate mood ${ }^{12}$. A German study of a larger series of 222 patients showed that nearly $80 \%$ of patients who changed their alcohol consumption habits reported an increase in smoking, but also in coffee, chocolate and sweet foods consumption ${ }^{13}$. Thus, it seems that decreasing or stopping alcohol consumption may be associated with nutritional problems involving several types of foods, but particularly sweet products.

The main objective of our study conducted in conjunction with an animal study assessing similar items (Alaux-Cantin et al ${ }^{14}$, submitted in the same issue) was to assess a specific need for sweet foods in AUD patients for 6 weeks after alcohol withdrawal and to measure the incidence of the sugar craving increase. Our hypothesis was that only a subset of the patients would be concerned. 


\section{PATIENTS AND METHOD}

This was a prospective, monocentric, observational study performed in a university hospital addictions treatment centre.

\section{PATIENTS}

Patients were included consecutively provided they met the following criteria: diagnosis of AUD according to the DSM-5; admission for alcohol withdrawal for 7 days in the short-stay unit and then in for 6 weeks in the rehabilitation unit; completely alcohol-free throughout the hospital stay assessed by use of an alcohol breath analyser. The non-inclusion criteria were: use of a drug other than alcohol or tobacco; severe co-morbid neurologic or psychiatric disease; Alzheimer's disease; severe cognitive deficits.

\section{METHOD}

Socio-demographic data (age, sex, education level, marital status), addiction profile (severity of addiction using the DSM-5), age of AUD onset, consumption of alcohol (g/d) before admission, smoking status and cognitive status using the Montreal Cognitive Assessment (MoCA) ${ }^{15}$ were recorded once on the first day of admission (except for the MoCA test which was administered after 7 to 10 days when withdrawal symptoms had disappeared).

All the parameters related to alcohol craving, sugar craving and tobacco consumption (number of cigarettes smoked per day) were recorded on the day after admission, referred to as Day 1 , and then on Day 15 and Day 45, that is just before discharge. The HADS (Hospital Anxiety and Depression Scale) was assessed on D1 and D45.

\section{Alcohol craving}

Alcohol craving was assessed using the French version of the obsessive-compulsive drinking scale for craving $^{16}$ of which score ranges from 0 to 40 . Patients were also asked to measure their craving using a visual analogue scale with scores ranging from 0 to 10 . Craving was explained to the patients as the perceived need to drink and/or the occurrence of obsessive thoughts about alcohol. 


\section{Sugar craving}

As there is no specific questionnaire to assess sugar appetence and craving, we used the following tools on D1, D15 and D45: (1) a visual analogue scale with scores ranging from 0 (no sugar craving at all) to 10 (very strong desire for sugar); (2) a visual assessment by the physician of the amount of sweet products stored (candies, chocolate, cakes, sweet drinks....) in the patients' rooms scored as follows: no stock $=0$; stock only for the current day $=1$; moderate stock for more than one day $=2$; big stock for several days $=3$; (3) assessment through a structured interview of the mean quantity of sweet products eaten and drunken on D15 and D45, expressed in g/day, and the reasons of consumption: lack, hunger, worry, wanting ; (4) weight.

\section{Food craving}

Food craving was assessed using the French version of the Food Cravings Questionnaire-Traitreduced (FCQT-r) $)^{17}$. It has been shown that this short self-administered questionnaire (15 items) can reliably assess food craving and monitor changes ${ }^{18}$. Each item is scored from 1 to 6 , producing a total score ranging from 15 to 90 . The higher the score, the stronger the craving. According to published data, a score equal to or higher than 50 has a sensitivity of $87.2 \%$ and a specificity of $75.2 \%$ for the detection of food addiction ${ }^{17}$.

\section{STATISTICAL ANALYSIS}

Quantitative variables are described as means \pm SDs and qualitative data as frequencies. To define an increase in the sugar craving score we used the method proposed by Jacobson $(1991)^{19}$. We calculated the reliable change index as the mean difference between the sugar craving scores at D1 and D45 divided by the standard error of the differences between the two scores. In order to compare the variation in sugar and alcohol craving, we calculated the difference between the respective scores (sugar minus alcohol craving score) at each investigation time and performed a linear regression according to time. Comparisons of quantitative parameters between groups with and without sugar craving were performed using a non-parametric test (Wilcoxon). Time data recorded on D1, D15 and D45 were analysed in each group using repeated measures ANOVA. A Bonferroni correction was applied to all tests. All analyses were performed using SPSS software V22.0 (IBM SPSS Inc, Armonk, NY, USA). 


\section{RESULTS}

During the study period, 40 patients met the inclusion criteria. Two patients voluntarily left the hospital 10 days after admission and 3 left on day 30. Their socio-demographic characteristics did not differ from those who remained in the study. The final sample therefore comprised 35 patients including 23 men and 12 women aged $54.0 \pm 9.8$ years. Their main socio-demographic characteristics are shown in Table 1.

On D1, the mean visual analogue score for sugar craving was 1.2 \pm 2.2 . It was null to low (0-1) in 27 patients $(77.1 \%)$, moderate $(2-4)$ in 4 patients $(11.4 \%)$ and strong $(\geq 5)$ in 4 patients $(11.4 \%)$. The

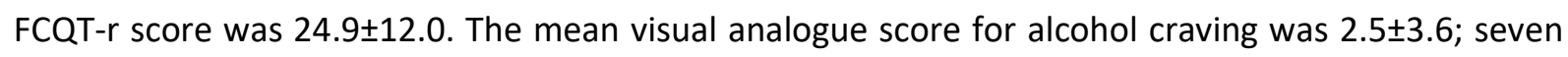
patients (22.6\%) had a score higher than 5. The mean OCDS score was $25.2 \pm 8.1$.

\section{Changes in craving over time}

The value of the reliable change index was 0.89 . In order to avoid any overestimation of the change, we decided to consider an increase in craving as a change equal or more than 2 points.

During the hospital stay, sugar craving increased by at least 2 points in 14 of the 35 patients (40\%). In 10 patients, the craving increase occurred between Day 1 and D15 and in the other 4, between D15 and D45.

Patients were then divided into 2 groups, one in which they presented an increase in sugar craving (Sugar Craving, SC, n=14) and one in which they presented no increase in sugar craving (No Sugar Craving, NSC, $n=21$ ). In the first group, that the sugar craving score rose from $0.1 \pm 0.3$ on D1 to $3.1 \pm 0.67$ on D15 $(p<0.05)$ and to $3.5 \pm 2.9$ on D45 ( $p<0.05$ vs D1) while it did not change in the second group (1.9 \pm 2.7 on D1 to $1.2 \pm 2.1$ on D45) (Fig 1 ).

In the SC group, storage of sweet products increased significantly $(p<0.02)$ from D1 to D15 $(0.57 \pm 0.23$ to $1.5 \pm 0.27)$ and still remained high on $\mathrm{D} 45(1.28 \pm 0.27, \mathrm{p}+0.03$ vs $\mathrm{D} 1)$ while no change was observed in the NSC group $(0.66 \pm 0.19$ on D1 to $0.90 \pm 0.21$ on D45) (Table 2). There were no significant differences observed in the reported sugar consumption nor in the FCQT-r scores on D15 and D45 \%) at D15 than (Table 2). In the SC group the mean reasons for eating sugar were: wanting $(42.9 \%)$ and hunger (35.7\%) then wanting (57.1\%) and worry (21.4\%) at D45. 
In both groups, the alcohol craving score (Fig 1) and the number of cigarettes smoked per day (Table 2 ) decreased significantly $(p<0.01)$ and similarly from D1 to D45. Plotting the values of the difference between the sugar and alcohol craving score according to time led to regression coefficient $\left(R^{2}\right)$ equal to $0.68(p<0.01)$ (data not shown).

While weight remained stable in the NSC group, it increased moderately but significantly in the SC group from $66.4 \pm 13.7 \mathrm{~kg}$ on D1 to $68.1 \pm 12.8$ on D45 $(p<0.05)$ (Table 2$)$.

\section{Comparison between the SC and NSC groups}

Between-group comparative data are presented in Table 3. No differences were found between the SC and NSC patients as regards sex, age, education level, quantity of alcohol consumed before admission for withdrawal, duration of excessive drinking, age of AUD onset, severity of AUD assessed with the DSM-5, OCDS score, anxiety and depression assessed with the HADS score, weight and cognitive function assessed with the MoCA test

Furthermore, on D1, the patients in the SC and NSC groups did not differ significantly in terms of reported amounts of sugar eaten per day, sweet products stored in their rooms, or alcohol craving score (Table 2). However, the FCQT-r score was significantly lower in the SC group than in the NSC group (19.6 \pm 7.0 vs $27.3 \pm 13.2, p<0.01)$. 


\section{DISCUSSION}

The objective of our study was to confirm a specific increase in the consumption of sweet products during alcohol withdrawal and to compare changes in craving for sweet products and alcohol. Assessing craving seemed relevant as it represents a central criterion of the addiction concept ${ }^{20}$.

This human study was conducted in parallel with another study in rats using the alcohol postdependent model in which the transfer of alcohol consumption to sugar was also evaluated during withdrawal. In hospitalized patients, given that alcohol abstinence is strictly controlled and access to sweet products inside the hospital is easy (ad libitum), the methodology was comparable to the preclinical one used by Alaux-Cantin et al. ${ }^{14}$ and led to several important observations.

Firstly, the majority of patients did not describe a craving for sugar before stopping alcohol. However, after alcohol withdrawal, the craving for sweet products increased significantly in a subgroup of patients during the first 2 weeks after withdrawal and continued to increase, but to a lesser extent, until the assessment on D45. To avoid overstating the incidence of these events, we increased by one point the threshold for detection of an increase in sugar craving. Sugar craving was observed in $14 / 35$ of the included patients (40\%) during hospitalization. Although previous research has shown that AUD patients like sweet tastes ${ }^{21}$, our data reveal that this effect tends to emerge during alcohol withdrawal, shedding light to a potential methodological drawback as recently pointed out ${ }^{22}$. It is especially very interesting to note that in the SC group converse changes were observed in sweet food craving and alcohol craving. Indeed, the latter, while moderately high on D1, continued to decrease over time and similarly in patients with and without sugar craving when measured after the initiation of alcohol withdrawal.

The question that must be asked is whether the increase in sugar craving is specific or simply a reflection of an increase in food intake. Three results were in favour of a specific increase in sweet products. Firstly, only patients who presented an increase in sugar craving stored significantly more sweet products in their rooms. It is important to note that the purchase of various sweet products is possible inside the hospital (shop and vending machines). This mimics the ad libitum access to sugar in the rodent model of Alaux-Cantin et al. ${ }^{14}$ Secondly, only patients in the SC group presented significant, although moderate, weight gain with no changes being observed in the NSC group. The observed weight gain therefore appears to be primarily related to the intake of sweet products, 
especially since an increase in storage of sweet products was only observed in the rooms of SC patients. Thirdly, the most frequent reason for eating sugar in the SC group was increased wanting.

An important element is the absence of relation between the increase in sugar craving and changes in tobacco consumption, which would of course constitute a major bias. Since patients consumed no other psychoactive products, the observed effect can therefore only be related to alcohol withdrawal. However why a sugar craving occurs in some patients remains undetermined. The severity of the AUD (number of DSM criteria) was not a predictive factor. Patients with SC had a lower MoCA at admission, suggesting that lower cognitive skills could generate increased susceptibility to craving, but the difference in MoCA score were not statistically significant. While the Hamilton scores were equally low at baseline in both groups with and without sugar craving, one cannot exclude the possibility that the former group experienced more anxiety and depression after alcohol withdrawal that they could try to alleviate by sugar intake ${ }^{23}$.

Our results confirm previously published findings that food consumption, particularly of sweet products, occurs after drug cessation ${ }^{12}$. Then again, it would have been very surprising if the results observed in humans were totally different from those obtained in preclinical models ${ }^{24}$ insofar as the reward neurocircuitries are very similar.

The limit of our study derives from the lack of a specific validated questionnaire to quantitatively assess sugar consumption and a craving score similar to the OCDS for alcohol. As the sweet products also often contain fat and/or protein we cannot be sure that the craving only concerns sugar. Although sugar craving was rather common, our series was too small to identify predictive factors and studies on larger samples are needed. Finally, patients were observed for an extended period, 6 weeks, but they were confined to a hospital and sugar appetence could well change after discharge.

In conclusion, increased intake and craving for sugar after alcohol withdrawal were observed in half of the patients included in our prospective study and similar results were obtained during withdrawal in a relevant model of AUD. Whether the substitution from alcohol to sugar corresponds to simple compensation or a true dependence transfer could not be specified and further studies are required. 
Author contributions:

P.P., S.A., B.N. were responsible for the study concept and design; R.A., M.T., P.S., A.L. were responsible for data collection; B.N., M.N., R.T. performed the data analysis; P.P., B.N. wrote the paper. All authors critically reviewed the content and approved the final version for publication. 


\section{REFERENCES}

1. Brousse G, Bendimerad P, de Chazeron I, Llorca PM, Perney P, Dematteis M. Alcoholism risk reduction in France: a modernised approach related to alcohol misuse disorders. Int J Environ Res Public Health. 2014; 11: 11664- 11675.

2. Krahn D, Grossman J, Henk H, Mussey M, Crosby R, Gosnell B. Sweet intake, sweet-liking, urges to eat, and weight change: relationship to alcohol dependence and abstinence. Addict Behav. 2006; 31: 622-631.

3. Colantuoni C, Rada P, McCarthy J, Patten C, Avena NM, Chadeayne A, Hoebel BG. Evidence that intermittent, excessive sugar intake causes endogenous opioid dependence. Obes Res. 2002; 10: 478- 488.

4. Fortuna JL. Sweet preference, sugar addiction and the familial history of alcohol dependence: shared neural pathways and genes. J Psychoactive Drugs. 2009; 42: 147- 151.

5. Volkow ND, O'Brien CP. Issues for DSM-V: should obesity be included as a brain disorder? Am J Psychiatry. 2007; 164: 708- 710

6. Koob GF, Volkow ND. Neurocircuitry of addiction. Neuropsychopharmacology. 2010; 35: 217238.

7. Gearhardt AN, White MA, Masheb RM, Grilo CM. An examination of food addiction in a racially diverse sample of obese patients with binge eating disorder in primary care settings. Compr Psychiatry. 2013; 54: 500- 505.

8. Kampov-Polevoy AB, Ziedonis D, Steinberg ML, Pinsky I, Krejci J, Eick C, Boland G, Khalitov E, Crews FT. Association between sweet preference and paternal history of alcoholism in psychiatric and substance abuse patients. Alcohol Clin Exp Res. 2003; 27: 1929- 1936.

9. McCarty CA, Kosterman R, Mason WA, McCauley E, Hawkins JD, Herrenkohl TI, Lengua L. Longitudinal associations among depression, obesity and alcohol use disorders in young adulthood. Gen Hosp Psychiatry. 2009; 31: 442-450.

10. Treur JL, Boomsma DI, Ligthart L, Willemsen G, Vink JM. Heritability of high sugar consumption through drinks and the genetic correlation with substance use. Am J Clin Nutr. 2016; 104: 1144- 1150.

11. Blum K, Bailey J, Gonzalez AM, Oscar-Berman M, Liu Y, Giordano J, Braverman E, Gold M. Neurogenetics of reward deficiency syndrome (RDS) as the root cause of "addiction transfer": a new phenomenon common after bariatric surgery. J Genet Syndr Gene Ther. 2011; 2012: S2-001 
12. Cowan J, Devine C. Food, eating, and weight concerns of men in recovery from substance addiction. Appetite. 2008; 50: 33- 42.

13. Junghanns K, Veltrup C, Wetterling T. Craving Shift in Chronic Alcoholics. Eur Addict Res. 2000; 6: 64- 70 .

14. Alaux-Cantin S, Alarcon R, Audegond C, Simon O'Brien E, Nalpas B, Perney P and Naassila M. Sugar intake and preference in ethanol postdependent rats: relevance for clinical consideration? Submitted in Addiction Biology 2019

15. Alarcon R, Nalpas B, Pelletier S, Perney P. MoCA as a Screening Tool of Neuropsychological Deficits in Alcohol-Dependent Patients. Alcohol Clin Exp Res; 2015 ;39:1042-8.

16. Ansseau M, Besson J, Lejoyeux M, Pinto E, Landry U, Cornes M, Deckers F, Potgieter A, Ades J. A French translation of the obsessive-compulsive drinking scale for craving in alcoholdependent patients: a validation study in Belgium, France, and Switzerland. Eur Addict Res. 2000; 6: 51- 56.

17. Brunault $P$, El Archi $S$, Ballon N, Réveillère $C$, Barrault $S$. Validation de la version française du Food Cravings Questionnaire-Trait-reduced : un auto-questionnaire simple et de passation rapide pour mesurer le craving alimentaire. Annales Médico-psychologiques, revue psychiatrique. 2018; 176: 788- 795.

18. Meule A, Hermann T, Kluber A. A short version of the Food Cravings Questionnaire-Trait: the FCQ-T-reduced. Front Psychol. 2014; 5: 1- 10.

19. Jacobson NS, Truax P. Clinical Significance: A Statistical Approach to Denning Meaningful Change in Psychotherapy. Research Journal of Consulting and Clinical Psychology. 1991; 59: $12-19$

20. Skinner MD, Aubin HJ. Craving's place in addiction theory: contributions of the major models. Neurosci Biobehav Rev. 2010; 34: 606- 623.

21. Kampov-Polevoy AB, Garbutt JC, Janowsky DS. Association between preference for sweets and excessive alcohol intake: a review of animal and human studies. Alcohol Alcohol. 1999; 34: 386-95.

22. Cummings JR, Tomiyama AJ. Bidirectional Associations Between Eating and Alcohol Use During Restricted Intake. Current Addiction Reports 2018 https://doi.org/10.1007/s40429$\underline{018-0180-4}$

23. Tomiyama AJ, Finch LE, Cummings JR. Did that brownie do its job? Stress, eating, and the biobehavioral effects of comfort food. In: Scott RA, Kosslyn SM, editors. Emerging Trends in the Social and Behavioral Sciences. Hoboken, NJ, USA: John Wiley \& Sons, Inc.; 2015.

24. Lenoir M, Serre F, Cantin L, Ahmed SH. Intense sweetness surpasses cocaine reward. PLoS One. 2007; 2: e698. 
Table 1: Main socio-demographic data

\begin{tabular}{lcc}
\hline $\mathbf{N}$ & 35 \\
Sex (M/W) & $23 / 12$ \\
Age & $54.0 \pm 9.8$ \\
Age of AUD onset & $32.5 \pm 12.9$ \\
Years of excessive drinking & $21.5 \pm 13.8$ \\
Alcohol (g/d) & $183 \pm 97$ \\
Smoker (\%) & 77.1 \\
Education (\%) & & \\
& $\leq \mathbf{1 2}$ & 88.6 \\
Weight & $>\mathbf{1 2}$ & 11.4 \\
BMI & & $73.6 \pm 16.7$ \\
BMI class (\%) & & $25.6 \pm 5.1$ \\
& & \\
& $\leq \mathbf{2 5}$ & 51.4 \\
OCDS & $\mathbf{2 5 < \leq 3 0}$ & 31.4 \\
MoCA & $>\mathbf{3 0}$ & 17.1 \\
HADS & & $25.2 \pm 8.1$ \\
& & $23 \pm 3.4$ \\
& & \\
& Mood & $5.7 \pm 2.4$ \\
& Anxiety & $10.5 \pm 4.3$ \\
& Depression & $9.8 \pm 3.5$ \\
\hline
\end{tabular}


Table 2: Changes over time in parameters (Mean $\pm S D$ ) associated with craving.

\begin{tabular}{|c|c|c|c|c|c|c|}
\hline & \multicolumn{3}{|c|}{ Sugar Craving group ( $N=14)$} & \multicolumn{3}{|c|}{ No Sugar Craving group ( $\mathrm{N}=\mathbf{2 1}$ ) } \\
\hline & Day 1 & Day 15 & Day 45 & Day 1 & Day 15 & Day 45 \\
\hline Sugar craving & $0.1 \pm 0.3$ & $3.1 \pm 2.6^{1}$ & $3.5 \pm 2.9^{1}$ & $1.9 \pm 2.7$ & $1.4 \pm 2.5$ & $1.2 \pm 2.1$ \\
\hline FCQT-r & $19.6 \pm 7.0^{4}$ & $22.5 \pm 8.6$ & $22.2 \pm 11.2$ & $27.3 \pm 13.2$ & $21.8 \pm 9.7$ & $22.2 \pm 11.2$ \\
\hline Stored sugar & $0.6 \pm 0.8$ & $1.5 \pm 1.0^{2}$ & $1.3 \pm 1.0$ & $0.7 \pm 0.8$ & $1.0 \pm 0.8$ & $0.9 \pm 0.9$ \\
\hline Q sugar $/ d$ & $11.2 \pm 7.1$ & $15.2 \pm 10.9$ & $12.8 \pm 13.3$ & $9.4 \pm 7.1$ & $11.7 \pm 15.1$ & $7.3 \pm 9.1$ \\
\hline Alcohol craving & $2.1 \pm 2.5$ & $1.0 \pm 1.4$ & $0.6 \pm 1.2^{1}$ & $2.7 \pm 3.4$ & $0.7 \pm 1.6^{3}$ & $0.7 \pm 1.7^{3}$ \\
\hline Weight & $66.4 \pm 13.3$ & $66.9 \pm 13.0$ & $68.1 \pm 12.3^{1}$ & $78.4 \pm 17.3$ & $78.8 \pm 17.1$ & $78.5 \pm 15.6$ \\
\hline $\mathrm{Cig} / \mathrm{d}$ & $24.9 \pm 12.8$ & $17.1 \pm 9.6$ & $12.0 \pm 8.2^{3}$ & $27.8 \pm 19.4$ & $19.9 \pm 15.2$ & $16.6 \pm 15.6^{3}$ \\
\hline
\end{tabular}

${ }^{1} p<0.05$ vs D1; ${ }^{2} p<0.02$ vs D $1 ;{ }^{3} p<0.01$ vs D1; ${ }^{4} p<0.01$ vs D1 No Sugar Craving 
Table 3: Comparison of baseline parameters between groups with sugar craving (SC) or without an increase in sugar craving (NSC)

\begin{tabular}{lccc}
\hline & SC & NSC & $\mathbf{p}$ \\
\hline Age & $55.1 \pm 9.1$ & $53.3 \pm 10.2$ & $\mathrm{NS}$ \\
Education (years) & $9.9 \pm 3.3$ & $9.8 \pm 3.2$ & $\mathrm{NS}$ \\
MoCA score & $21.6 \pm 3.8$ & $24 \pm 2.9$ & $\mathrm{NS}$ \\
DSMV ( $\boldsymbol{n}$ item) & $8.4 \pm 2.2$ & $7.7 \pm 2.8$ & $\mathrm{NS}$ \\
Age AUD begining & $37.8 \pm 12.6$ & $29 \pm 12.2$ & $\mathrm{NS}$ \\
Duration of AUD & $17.4 \pm 11.6$ & $24.3 \pm 14.8$ & $\mathrm{NS}$ \\
Q_alcohol/d & $196.8 \pm 124.3$ & $174.8 \pm 77.2$ & $\mathrm{NS}$ \\
Weight & $66.4 \pm 13.3$ & $78.4 \pm 17.3$ & $\mathrm{NS}$ \\
HAD anxiety & $10.6 \pm 3.8$ & $10.5 \pm 4.7$ & $\mathrm{NS}$ \\
HAD depression & $9.9 \pm 3.3$ & $9.8 \pm 3.8$ & $\mathrm{NS}$ \\
OCDS at D1 & $25.1 \pm 7.7$ & $25.2 \pm 8.6$ & $\mathrm{NS}$ \\
\hline
\end{tabular}


Figure 1

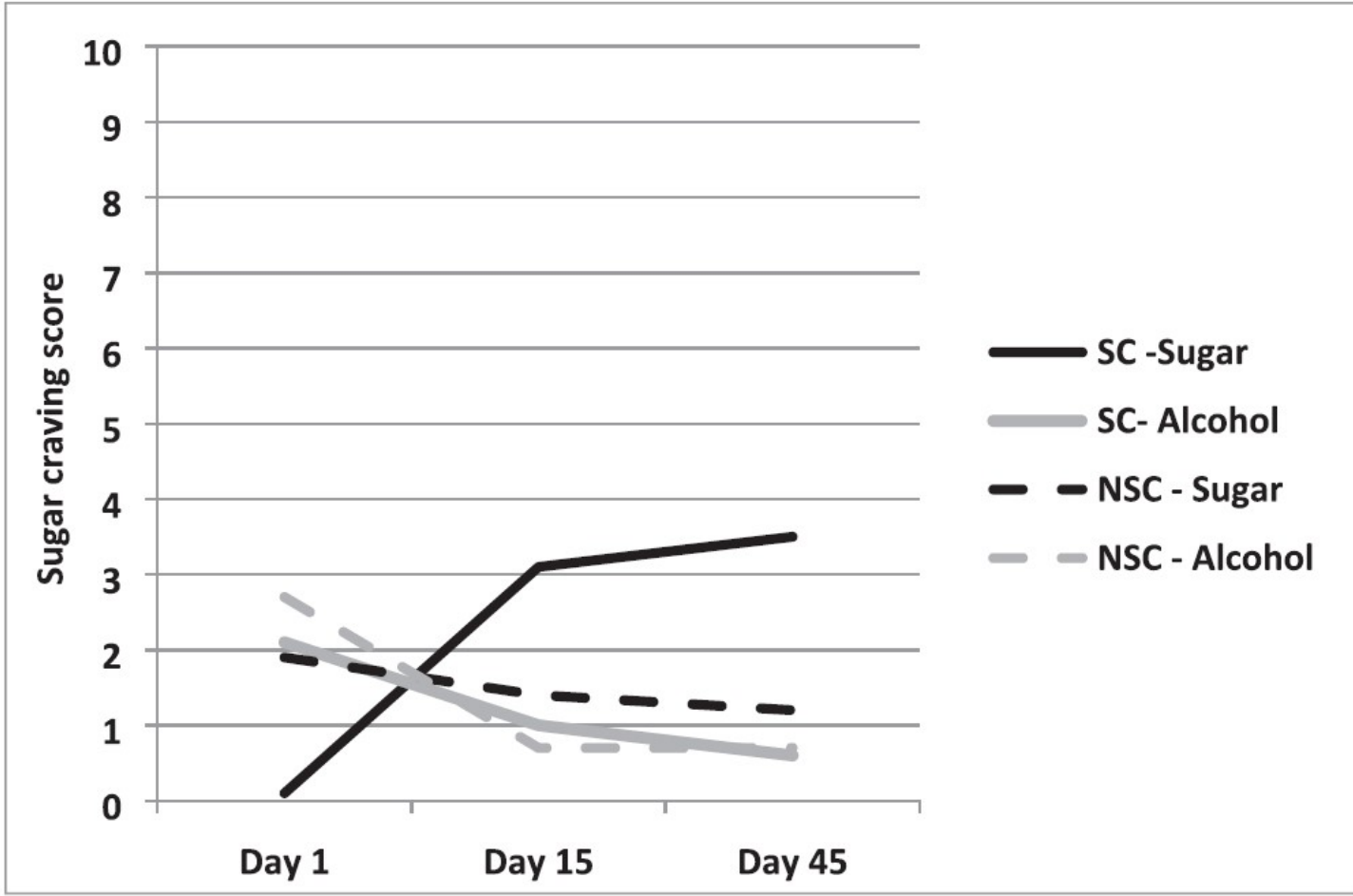

FIGURE 1 Changes over time in sugar and alcohol craving in the SC and NSC groups 
Figure 2

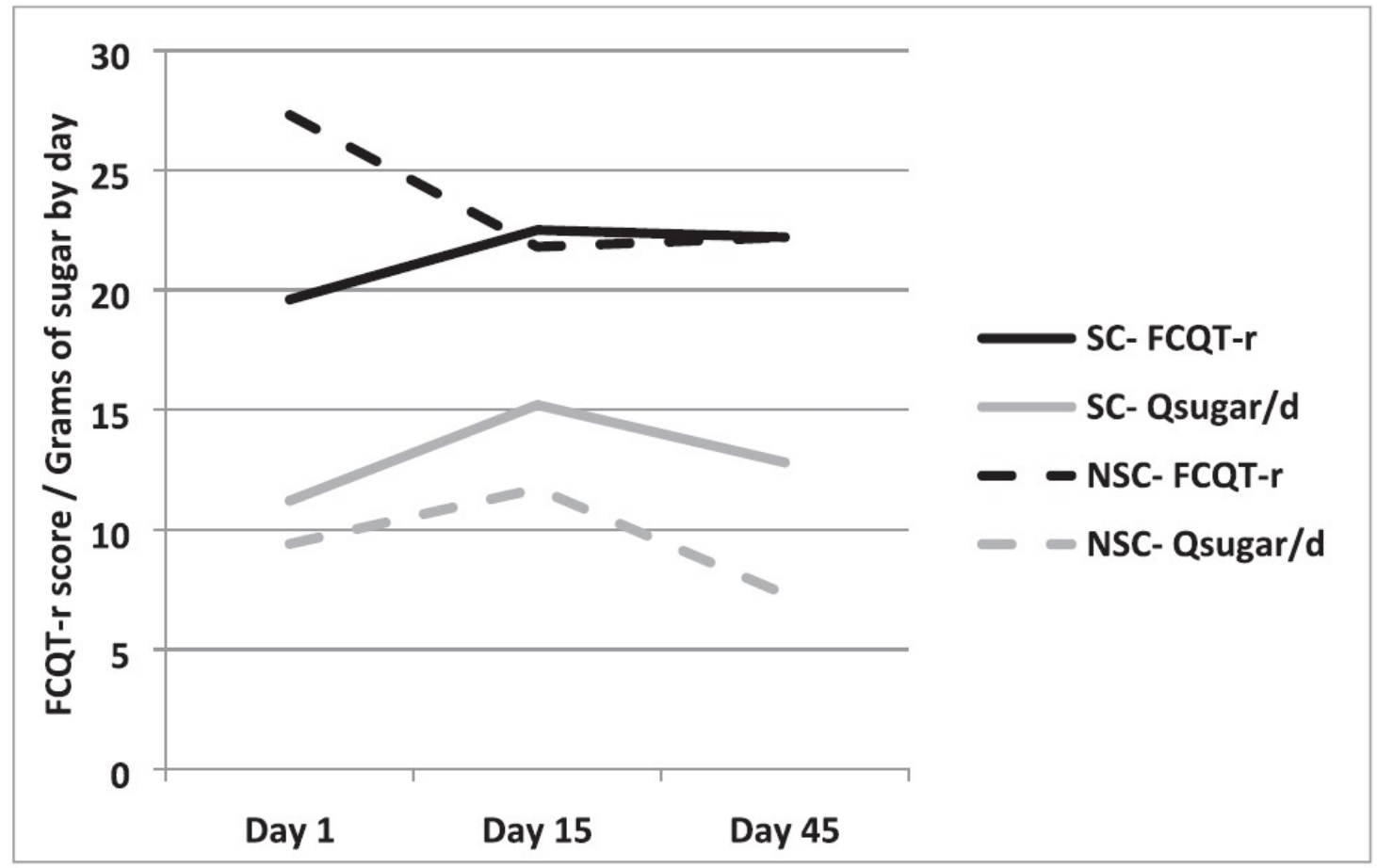

FIGURE 2 Changes over time in the FCQT-r score and quantity of sugar eaten per day in the SC and NSC groups 